\title{
The $\pi^{+} \pi^{-} \pi^{0}$ final state in two-photon collisions at Belle
}

\section{S. Hou* for the Belle Collaboration}

National Taiwan University, Taipei 106, Taiwan

E-mail: suen@bmail.kek.jp

ABSTRACT: Resonance formation in two-photon collisions in the $\pi^{+} \pi^{-} \pi^{0}$ final state was investigated. The spin-parity parameters were determined by fitting the data with Monte Carlo generated samples for the $a_{2}(1320)$ meson and a resonance at $1750 \mathrm{MeV} / \mathrm{c}^{2}$ with various partial wave hypotheses. The $a_{2}(1750)$ meson is observed and found to be predominantly in the helicity 2 state. Its mass, total width and two-photon radiative width were also measured.

The production of the exclusive $\pi^{+} \pi^{-} \pi^{0}$ final state in two-photon collisions is dominated by the formation of the $a_{2}(1320)$ meson in a predominantly helicity 2 state. In addition, a resonance state in the mass region around $1750 \mathrm{MeV} / \mathrm{c}^{2}$ has been reported by many experiments [1]. The L3 result [2] indicates a new radial excitation state, $a_{2}(1750)$, of predominant $J^{P}=2^{+}$, helicity 2 wave with $\rho \pi$ and $f_{2} \pi$ intermediate states. In this report, we present the preliminary Belle result based on a $6.54 \mathrm{fb}^{-1}$ data sample collected during the year 2000 at a center-of-mass energy $\sqrt{s}=10.58 \mathrm{GeV}$.

The $\pi^{+} \pi^{-} \pi^{0}$ final state is produced via two body intermediate states with the allowed values of the orbital angular momentum between the di-pion isobar and the third pion. The $a_{2}(1320)$ meson is known to decay through $\rho^{ \pm} \pi^{\mp}$ states. In the mass region corresponding to the $a_{2}(1750)$ meson, the spin-parity to be considered are $J^{P}=0^{-}, 2^{-}$and $2^{+}$with the decays in the $\pi^{+} \pi^{-} \pi^{0}$ phase space distribution and the $\rho^{ \pm} \pi^{\mp}$ and $f_{2} \pi^{0}$ intermediate states. The photon flux for the Monte Carlo simulation is calculated for the two-photon luminosity function [3]. The decay amplitude is a sum of orthogonal spherical harmonics multiplied by the di-pion Breit-Wigner terms. The interference between $\rho \pi$ and $f_{2} \pi$ is present for $J^{P}$ $=2^{+}$. The decay matrix element is given by

$$
\mathcal{T}=\sum_{\lambda} R_{\lambda}\left|\left(\frac{T_{\lambda}\left(\rho^{+} \pi^{-}\right)}{m_{\rho}^{2}-s_{\pi^{+} \pi^{0}}-i m_{\rho} \Gamma_{\rho}}+\frac{T_{\lambda}\left(\rho^{-} \pi^{+}\right)}{m_{\rho}^{2}-s_{\pi^{-} \pi^{0}}-i m_{\rho} \Gamma_{\rho}}\right)+\alpha \exp (i \phi) \frac{T_{\lambda}\left(f_{2} \pi^{0}\right)}{m_{f_{2}}^{2}-s_{\pi^{+} \pi^{-}}-i m_{f_{2}} \Gamma_{f_{2}}}\right|^{2},
$$

where $R_{\lambda}$ is the relative helicity density, $\alpha^{2}$ is the ratio of the branching ratio of $f_{2} \pi$ and $\rho \pi$, and $\phi$ is the relative phase angle of the two decay amplitudes. The angular distributions for the di-pions and the pions from di-pion decay are described by the transition amplitude $T_{\lambda}$.

\footnotetext{
${ }^{*}$ Speaker.
} 
The Belle experiment [4] was designed for data taking in a high luminosity environment. As a compensation to the high event rate, the prior event selection for two-photon events was imposed with a strict momentum threshold of $p_{t}>300 \mathrm{MeV} / c$. Two-photon events were selected for the total energy sum of observed tracks and photons smaller than $5 \mathrm{GeV}$. Charged pions were detected by the central drift chamber and the silicon vertex detector in a $1.5 \mathrm{~T}$ field. The photons from $\pi^{0}$ decay were detected by the electromagnetic calorimeter of CsI crystals. To eliminate contamination from beam related background, photon energy was required to be greater than $150 \mathrm{MeV}$. The mass resolution for $\pi^{0}$ (Fig. 1.a) is $6 \mathrm{MeV} / \mathrm{c}^{2}$. $\pi^{0}$ candidates were selected for the invariant mass of photon pair within three-sigma of the $\pi^{0}$ mass.

The photons from the $\pi^{0}$ decay have large opening angle $(\Omega)$ between them and to the charged pions. Background events tend to have smaller opening angle distributions and were excluded by $(\min \Omega(\pi \gamma)+2 \cdot \Omega(\gamma \gamma))>1 \operatorname{Rad}$ in the laboratory frame and $(\min \Omega(\pi \gamma)+$ $\Omega(\gamma \gamma))>1 \mathrm{Rad}$ in the $\pi^{+} \pi^{-} \pi^{0}$ rest frame. Events with di-pion masses outside of the Dalitz contour for the $\rho$ meson were also excluded by requiring $m\left(\pi^{+} \pi^{0}\right)+m\left(\pi^{-} \pi^{0}\right)>1 \mathrm{GeV} / \mathrm{c}^{2}$. The contamination of $\gamma \gamma \rightarrow \pi^{+} \pi^{-}$events may occur by an accidental coincidence with a $\pi^{0}$ reconstructed for a random combination of beam background. Such events were suppressed by requiring $p_{t}^{2}\left(\pi^{+} \pi^{-}\right)>0.01 \mathrm{GeV}^{2} / c^{2}$. The selection efficiency for $\pi^{+} \pi^{-} \pi^{0}$ events was estimated by Monte Carlo. Illustrated in Fig. 1.b is the selection efficiency versus mass for $a_{2}(1320)$ and $a_{2}(1750)$ of $J^{P}(\lambda)=2^{+}(2)$. The average selection efficiencies for $a_{2}(1320)$ and $a_{2}(1750)$ are $0.70 \%$ and $2.9 \%$, respectively. The trigger efficiency depends on the angular distribution of final state pions. In comparison, for $J^{P}(\lambda)=2^{+}(0)$, with more charged pions distributed in the barrel region, the selection efficiencies are $1.3 \%$ and $4.2 \%$, respectively.

The $p_{t}^{2}$ distributions of $\pi^{+} \pi^{-} \pi^{0}$ candidates are shown in Fig. 2 for the two mass regions corresponding to the formation of $a_{2}(1320)$ and $a_{2}(1750)$. The photon flux in the Monte Carlo was simulated with a $\rho$-pole form factor. The low $p_{t}^{2}$ events correspond to interactions of quasi-real photons with $Q^{2} \sim 0$, and are the least contaminated by beam background or other two-photon interactions. Therefore a stringent requirement of $p_{t}^{2}<0.0002 \mathrm{GeV}^{2} / c^{2}$ was applied for the final $\pi^{+} \pi^{-} \pi^{0}$ candidates. The invariant mass spectrum obtained is
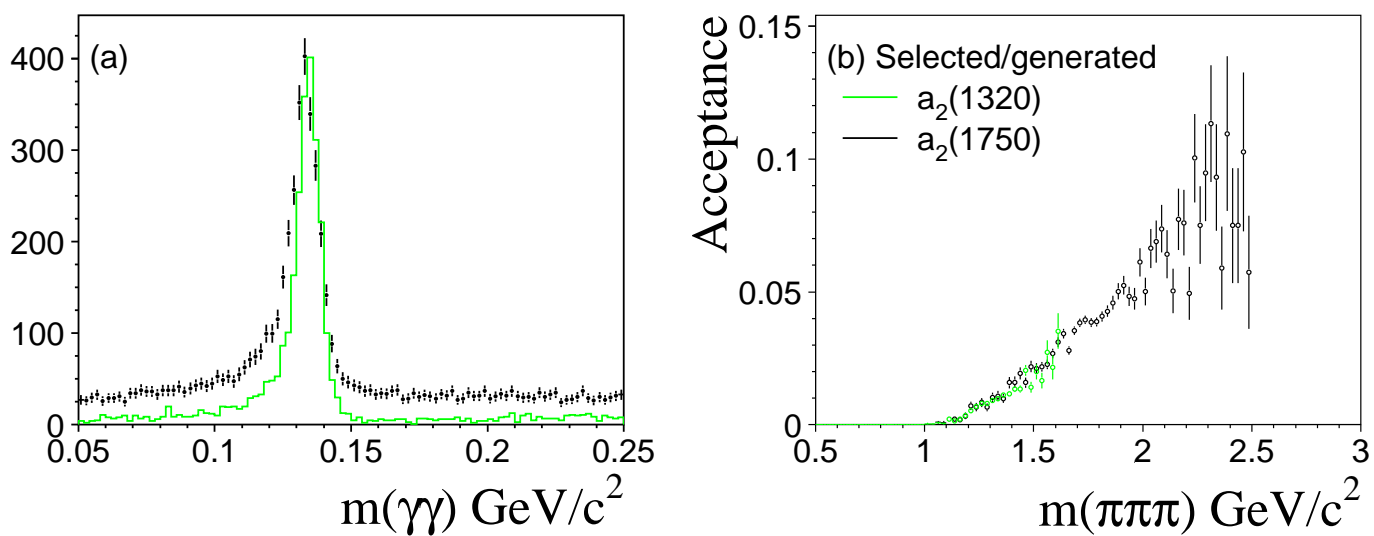

Figure 1: (a) Invariant mass of photon pairs, Monte Carlo is shown in histogram. (b) Selection efficiencies estimated by Monte Carlo for $a_{2}(1320)$ and $a_{2}(1750)$. 

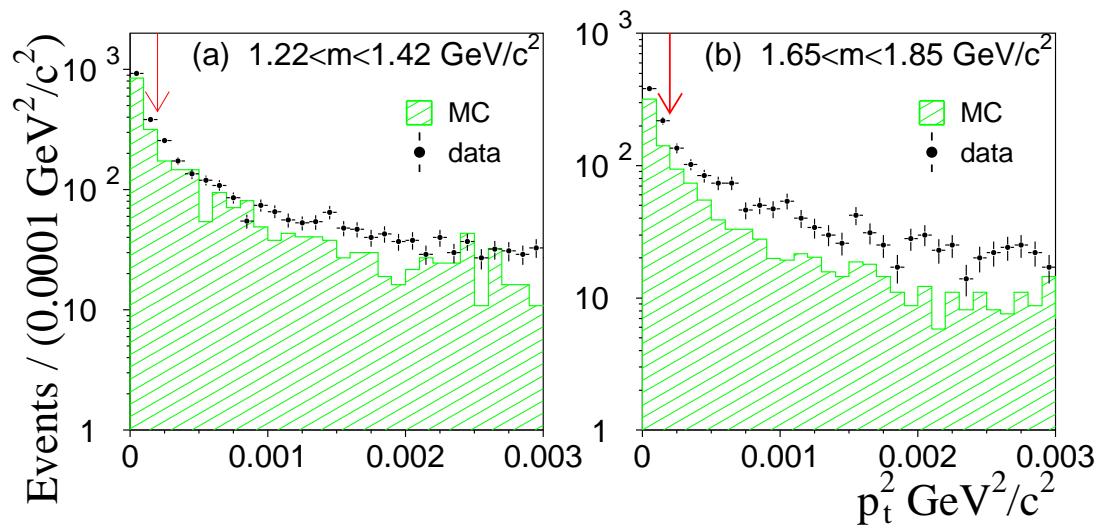

Figure 2: Distributions of transverse momentum squared $\left(p_{t}^{2}\right)$ for $\pi^{+} \pi^{-} \pi^{0}$ candidates in two mass ranges. The Monte Carlo simulated with a $\rho$-pole form factor are normalized to $\Gamma_{\gamma \gamma}=1.0 \mathrm{keV}$ for $a_{2}(1320)$ and $\Gamma_{\gamma \gamma} \cdot B R\left(\pi^{+} \pi^{-} \pi^{0}\right)=0.29 \mathrm{keV}$ for $a_{2}(1750)$.

shown in Fig. 3.a. The enhancement in data in the $1750 \mathrm{MeV} / \mathrm{c}^{2}$ region is clearly seen.

In this study we focus on the determination of the resonance parameters in the $1750 \mathrm{MeV} / \mathrm{c}^{2}$ region. The invariant mass spectrum of the data was fitted to the Monte Carlo distributions of $a_{2}(1320)$ and $a_{2}(1750)$ with the background parameterized by a second order polynomial. The Monte Carlo prediction for $a_{2}(1320)$ was normalized and fixed to the nominal two-photon radiative width and used as a side-band constraint for background. The background distribution was also constrained by the side-band of above $2.0 \mathrm{GeV} / \mathrm{c}^{2}$. The resonance mass and width were determined by minimizing the $\chi^{2}$ to the Monte Carlo distributions generated at several mass positions and widths. The $\chi^{2} /$ ndf obtained in the mass region of $1.55<m(3 \pi)<2.0 \mathrm{GeV} / \mathrm{c}^{2}$ is 1.3 . The background fraction of the fit is $39 \%$. The two-photon radiative width is obtained by normalizing the observed number of events to the selection efficiency. The dominant systematic uncertainties come from the estimation of background and selection efficiency. The effects were evaluated with the Monte Carlo distributions scaled for the errors in resonance mass, width and background
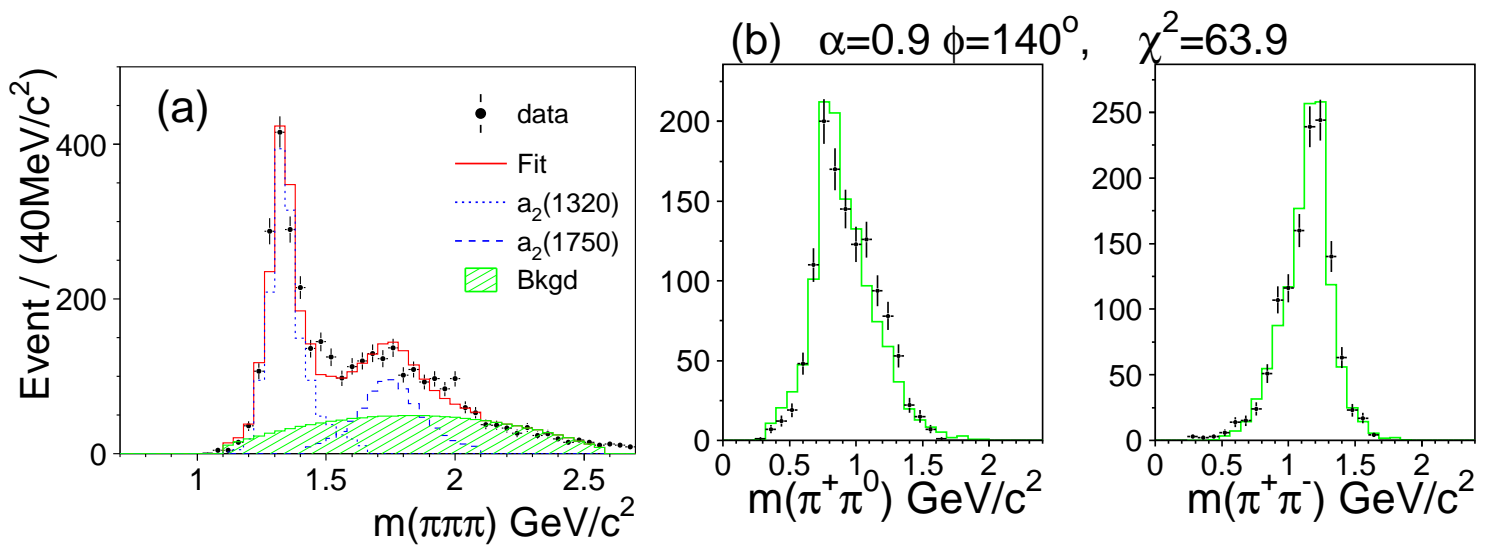

Figure 3: (a) Invariant mass distribution for $\pi^{+} \pi^{-} \pi^{0}$ candidates of $p_{t}^{2}<0.0002 \mathrm{GeV}^{2} / c^{2}$. (b) The di-pion mass spectra compared to the Monte Carlo of interference amplitude $\alpha=0.9$ and phase angle $\phi=140^{\circ}$. 
level. The selection efficiency differs for the decay intermediate state and spin-parity. For a pure $J^{P}=2^{+}$helicity 2 state with equal decay amplitude into the interference of $\rho \pi$ and $f_{2} \pi$, the resonance parameters obtained are

$$
\begin{array}{ll}
\text { mass } & m=1740 \pm 10 \pm 10 \mathrm{MeV} / \mathrm{c}^{2} ; \\
\text { width } & \Gamma=290 \pm 30 \pm 20 \mathrm{MeV} ; \\
\text { radiative width } & \Gamma_{\gamma \gamma} \cdot B R\left(\pi^{+} \pi^{-} \pi^{0}\right)=0.27 \pm 0.02 \pm 0.04 \mathrm{keV} .
\end{array}
$$

The invariant mass spectra of di-pions for the $a_{2}(1750)$ candidates were examined for the decay intermediate states. The interference of $\rho \pi$ and $f_{2} \pi$ for $J^{P}=2^{+}$is expressed by the phase angle $\phi$ and the relative amplitude $\alpha$. The phase angle has an effect of shifting the di-pion mass peaks of $\rho$ and $f_{2}$ toward each other as $\phi$ gets smaller. The peak positions are compatible to the nomial values at $\phi=180^{\circ}$. The two interference parameters were determined by $\chi^{2}$ tests to the Monte Carlo predictions of pure $J^{P}=2^{+}$helicity 2 state. Full simulations were performed for discrete phase angles from 90 to 240 degrees with amplitude from 0.8 to 1.2. Shown in Fig. 3.b is a test to the Monte Carlo of $\alpha=0.9$ and $\phi=140^{\circ}$. The phase angle determined by the $\chi^{2}$ tests is $125 \pm 5$ degree. The systematic uncertainty is mainly from the detection efficiency and simulation for di-pion and is estimated to be 10 degree. The relative amplitude obtained is $\alpha=0.9 \pm 0.1$ with a systematic error of 0.2 .

The resonance spin-parity can be distinguished by the magnitude of the norm of the decay plane. Shown in Fig. 4 are the distributions for an optimized $\Lambda$ parameter, $\Lambda=$ $\left|\vec{p}\left(\pi^{+}\right) \times \vec{p}\left(\pi^{-}\right) / Q\right|^{2}$, for the norm of decay plane normalized to the kinetic energy $Q$. The spectra for events in the $1320 \mathrm{MeV} / \mathrm{c}^{2}$ and the $1750 \mathrm{MeV} / \mathrm{c}^{2}$ regions were tested to Monte Carlo for decays into $\pi^{+} \pi^{-} \pi^{0}$ phase space, $\rho \pi$ and $f_{2} \pi$ of $J^{P}=2^{-}$, and the $\rho \pi$, $f_{2} \pi$ interference of $J^{P}=2^{+}$with helicity 0 and 2 . The $\chi^{2}$ values obtained (listed in the figures) are consistent with the $J^{P}=2^{+}$predictions.

The angular dependence of the spin-parity and helicity state can be directly observed in the polar angle distributions of the final state pions. The $\cos \theta$ distribution for $\pi^{0}$ and the difference in $\cos \theta$ for $\pi^{+}$and $\pi^{-}$are shown in Fig. 5 for events in the two mass regions and compared to Monte Carlo of $J^{P}=2^{+}$helicity 2 state. The asymmetric distribution observed for $\pi^{0}$ is due to the boost in the Belle asymmetric beam configuration

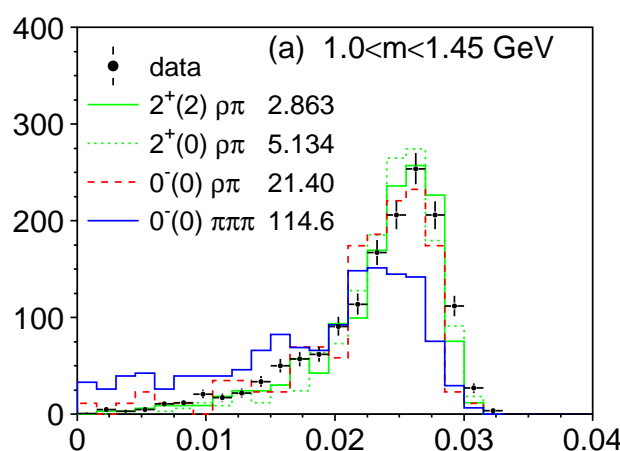

$\Lambda$

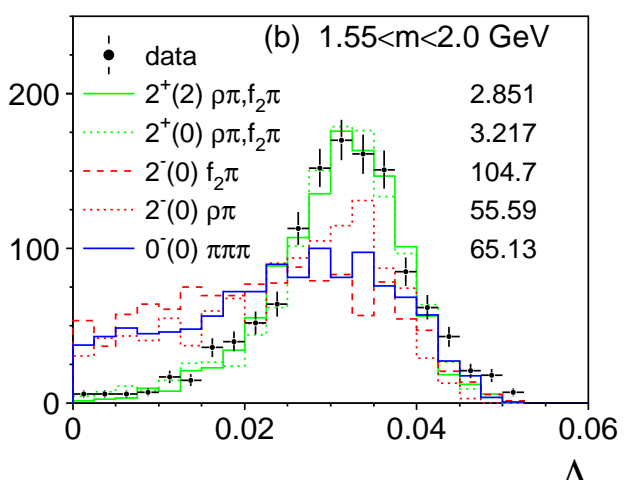

$\Lambda$

Figure 4: $\Lambda$ distributions and the $\chi^{2}$ tests to the Monte Carlo predictions for the spin-parity dependence for events in two mass regions. Monte Carlo distributions are normalized to data. 
(a) $1.0<\mathrm{m}<1.45 \mathrm{GeV}$

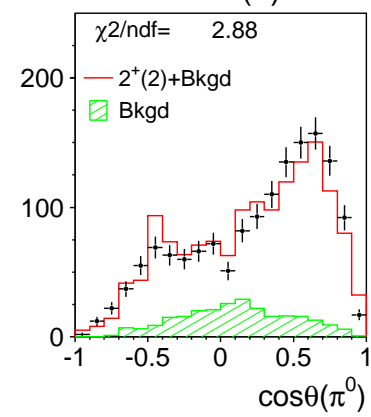

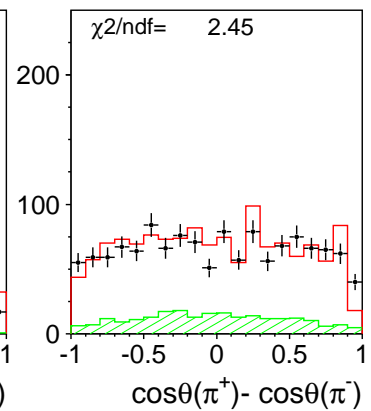
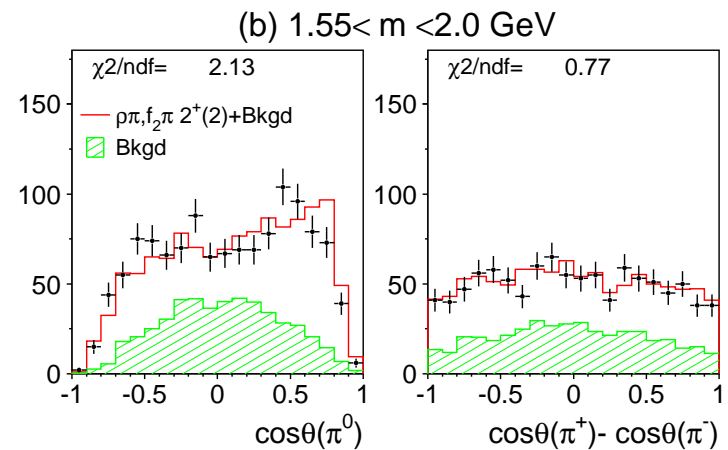

Figure 5: $\cos \theta$ distributions for final state pions in the resonance rest frame. Histograms are the Monte Carlo predictions for $J^{P}=2^{+}$, helicity 2. Background in $\pi^{+} \pi^{-} \pi^{0}$ phase space distribution is applied for an event fraction of $17 \%$ for $a_{2}(1320)$, and $39 \%$ for $a_{2}(1750)$.

\begin{tabular}{|c|c|c|c|c|}
\hline Mass & Mode & $J^{P}$ (helicity) & $\begin{array}{c}\chi^{2} / \mathrm{ndf} \\
\cos \theta\left(\pi^{0}\right)\end{array}$ & $\begin{array}{c}\chi^{2} / \mathrm{ndf} \\
\cos \theta\left(\pi^{+}\right)-\cos \theta\left(\pi^{-}\right)\end{array}$ \\
\hline \multirow[t]{2}{*}{$1320 \mathrm{MeV} / \mathrm{c}^{2}$} & $\rho^{ \pm} \pi^{\mp}$ & $2^{+}(0)$ & 66.3 & 12.1 \\
\hline & $\rho^{ \pm} \pi^{\mp}$ & $2^{+}(2)$ & 2.9 & 2.5 \\
\hline \multirow[t]{4}{*}{$1750 \mathrm{MeV} / \mathrm{c}^{2}$} & $\rho \pi$ & $2^{-}(0)$ & 14.5 & 2.7 \\
\hline & $f_{2} \pi^{0}$ & $2^{-}(0)$ & 7.8 & 11.8 \\
\hline & $\rho \pi, f_{2} \pi^{0}$ interference & $2^{+}(0)$ & 13.4 & 6.4 \\
\hline & $\rho \pi, f_{2} \pi^{0}$ interference & $2^{+}(2)$ & 2.1 & 0.8 \\
\hline
\end{tabular}

Table 1: $\chi^{2}$ tests for the $\cos \theta$ distributions. Events are selected in two mass ranges of 1.0 to $1.45 \mathrm{GeV} / \mathrm{c}^{2}$ and 1.55 to $2.0 \mathrm{GeV} / \mathrm{c}^{2}$, respectively.

and the energy threshold for photons. Background events are assumed to be distributed randomly, therefore distributions from the $\pi^{+} \pi^{-} \pi^{0}$ phase space simulations were applied and weighted for the fractions obtained from the mass spectrum fit. The distributions for the $1750 \mathrm{MeV} / \mathrm{c}^{2}$ mass region were also tested for $J^{P}=2^{-}$and decays into $\rho \pi$ or $f_{2} \pi$ states. The $\chi^{2}$ values obtained are listed in Table 1.

In this study the Monte Carlo samples were generated assuming independent resonance formations for the $a_{2}(1320)$ and a resonance at $1750 \mathrm{MeV} / \mathrm{c}^{2}$. The dominant $a_{2}(1320)$ events serve as a reference sample for studying the spin-parity of events in the $1750 \mathrm{MeV} / \mathrm{c}^{2}$ region. The di-pion mass spectra and the angular distributions of final state pions are consistent with the predictions for the $a_{2}(1750)$ of $J^{P}=2^{+}$helicity 2 wave with decays of $\rho \pi$ and $f_{2} \pi$ intermediate states.

\section{References}

[1] Particle data group, Euro. Phys. J. C15 (2000) 1, and references therein.

[2] L3 Collab., M. Acciarri et al., Phys. Lett. B 413 (1997) 147.

[3] V.M. Budnev et al., Phys. Rep. 15 (1975) 181.

[4] Belle Collab., K. Abe et al., KEK Progress Report 2000-4 (2000), to be published in Nucl. Inst. and Meth. A. 